\title{
Micro-scale Compression of Ni-based Metallic Glass
}

\author{
Bin WU ${ }^{a}$, Tao FENG ${ }^{b}$, Abdur RAUF and Chun-Yu GUO
}

\author{
Herbert Gleiter Institute of Nanoscience (HGI), Nanjing University of Science \& Technology \\ (NUST) Nanjing 210094, China \\ aBin.Wu@njust.edu.cn \\ btao.feng@njust.edu.cn
}

Keywords: Micro-scale, Compression, FIB, Nanoindentation.

\begin{abstract}
Mechanical properties of $\mathrm{Ni}_{80} \mathrm{P}_{20}$ metallic ribbon fabricated by melt spinning was tested in micro-scale compression, $1 \mu \mathrm{m}$ pillars produced by Foused Ions Beam (FIB) were characterized with nanoindentation. Stress-strain curve together with compressed pillar morphology analyses show that ribbon deformation mechanism may attribute to the shear transformation zones (STZs), which as preferential shear band nucleation sites to form shear bands. Quantitative micro-scale characterization of compression on stress-stain would be momentous significance.
\end{abstract}

\section{Introduction}

Metallic glasses (MGs) are the focus of outstanding properties for their unique structures bringing potential applications for decades[1-3]. Several advantages, such as excellent mechanical and magnetic properties make them appropriate for micro electro mechanical system (MEMS)[4]. Firstly the unique amorphous structure contains no imperfection likes defects and dislocations in crystalline materials, which always cause yielding failure. High strength and high hardness of MGs compared with other metallic materials are desirable for some special MEMS parts. High elastic strain appears before yielding together with great resilience is also necessary for MEMS application[5]. Comparing to crystalline boundaries between grains take shape multiphase mixtures, the conventional alloy structure will be prevailing. Corrosion resistance of MGs also is superior to crystalline alloys. First theoretical analysis of the amorphous iron was performed in 1925, and increasing properties of amorphous iron up to 1000 times comparing to the known ones measured in practice[6]. Considering all the advantages above, MGs are attractive as MEMS materials[7]. Up to now, size-dependent effects have been observed in mechanical properties tests[8-10], which are fulfilled by different kinds of techniques like nanoindentation, microtorsion, microbending. Not only in crystalline but also amorphous materials, miro-scale and nano-scale show different mechanical properties that are distinct with bulk materials in room temperature ${ }^{[11]}$. So quantitative micro-scale characterization of compression on stress-stain would be momentous significance.

Focused ion beam (FIB) with gallium as ion source. The FIB devices first developed in 1975, then the new technology is first applied in the semiconductor industry[12]. Liquid metal ion (gallium ion) applied as a current with high energy density beam to mill micro scale structure materials like integrated circuits. In the following years, FIB has priority in semiconductor for special sample preparation. Typical applications include quality control, chip repair and microelectronics fault analysis. Production quality control, chip repairs, and production failure analysis. Preparation of site-specific transmission electron microscope (TEM) specimen can be realized with the initiating advanced development of this technique in eighties and nineties. Some publication represent the progress: lift-out of desirable site materials foil and then mill the foil less than $100 \mathrm{~nm}$ to prepare a perfect TEM sample[13]. With the progress of science and technology, more FIB applications have been reported, some major challenges that conventional methods can't be realized are overcome by FIB such as micromachining of diamond, specimen preparation for TEM and three-dimensional atom probe tomography (APT), and 3D cross sectioning of materials[14]. The other excellence is the success ratio and quality is improved tremendously by FIB.The most important advantage for us is that we can use FIB to mill some pillars to test 
compression.

Nanoindentation testing has developed into a commonplace technique to characterize mechanical properties at small scale for film or other surface of materials[15]. With high resolution of recorder, load depth of indenter into the sample together with the measured load rapidly, analysis of the loaddisplacement data is used to explain experimental research of fundamental physic properties[16]. With the determinate contact area of the indenter, hardness of specimen is obtained easily, in the meantime corresponding young modulus, fracture toughness, hardening index, and yield strength also be obtained under the similar conditions. With high resolution of load-displacement curve, information about mechanical deformation is obvious. Correlative events like dislocation activation, initial instability of shear band, creep deformation can be detected by the nanoindentation[17]. Recently high temperature in-situ nanoindentation characterization is developed and in-situ imaging help to indented volume of indenter so more detail information correlate to phenomena will be present in front of us. The compression in this study is tested with a nanoindentation flat punch to obtain high resolution of load-displacement data.

\section{Experimental Part and Discussion}

Schematic of a cylindrical pillar and its micro-compression (made of the same material) is showed in Fig.1. Firstly, Ni-based metallic glass was prepared with the melt-spinning method, vertical pillar like an island on surface was fabricated, and then compression on the pillar is done to obtain loaddisplacement data. As shown in Fig.1 (a) metallic ribbon with composition $\mathrm{Ni}_{80} \mathrm{P}_{20}$ has been prepared by mixing of $99.99 \% \mathrm{Ni}$ and $\mathrm{P}$ power 3 times in vacuum induction melting. The prepared pallet of $\mathrm{Ni}_{80} \mathrm{P}_{20}$ alloy was emerged into quartz tube and then melted over roller spinning at 3500r/min in Ar environment with 0.5 Bar. The metallic ribbon were polished with $\mathrm{SiC}$ paper from \#1200 to \#1400, then ribbon surface was polished to mirror finished with a diamond polishing paste. Several micro columnar pillars (diameter about $1 \mu \mathrm{m}$ ) have been fabricated by ZEISS AURIGA FIB (Focus Ion Beam)/SEM (Scanning Electron Microscope) system. Milling process of fabrication as show in Fig.1 (b), first step rough milling with an initial high $\mathrm{Ga}$ ion beam with $30 \mathrm{KeV}$ and $16 \mathrm{nA}$ to dig a ring-shaped pit, left a island in the center, then reduce the machining beam current to $20 \mathrm{pA}$ gradually, $1 \mu \mathrm{m}$ pillar which has an aspect ratio $(\mathrm{h} / \mathrm{d}) \sim 3.5$ with a taper angle of $3^{\circ}$ is accomplished finally. Compression test shows in Fig.1(c) has been performed under displacement rate controlled mode at a nominal strain rate of $10^{-3} \mathrm{~s}^{-1}$ with the diamond flat punch of Agilent Nano Indenter G200. Pillars prior to compression and compressed morphology are observed by scanning electron microscopy (SEM, 5KV).

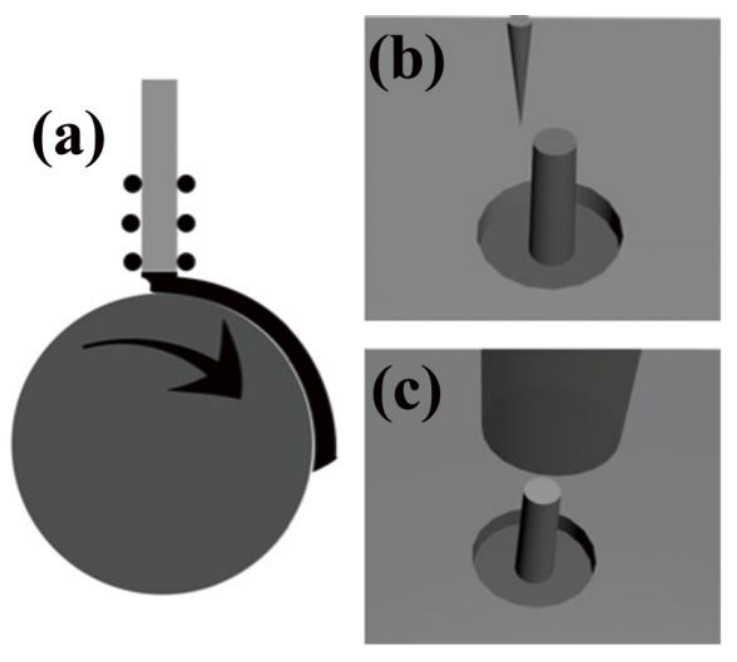

Fig. 1 schematic of the preparation process. (a) melt spinning of $\mathrm{Ni}_{80} \mathrm{P}_{20}$, (b) micro-scale pillar fabricated by FIB, (c) compression test by nanoindentation. 
Microstructural characterization has been performed with the help of X-ray diffraction (XRD, $\mathrm{Cu}$ $\mathrm{K} \alpha$ ). XRD pattern of metallic glass is displayed in Fig. $2,2 \theta$ from $35^{\circ}$ to $60^{\circ}$ shows a typical broad diffraction pattern indicating amorphous phase of $\mathrm{Ni}_{80} \mathrm{P}_{20}$ metallic glass.

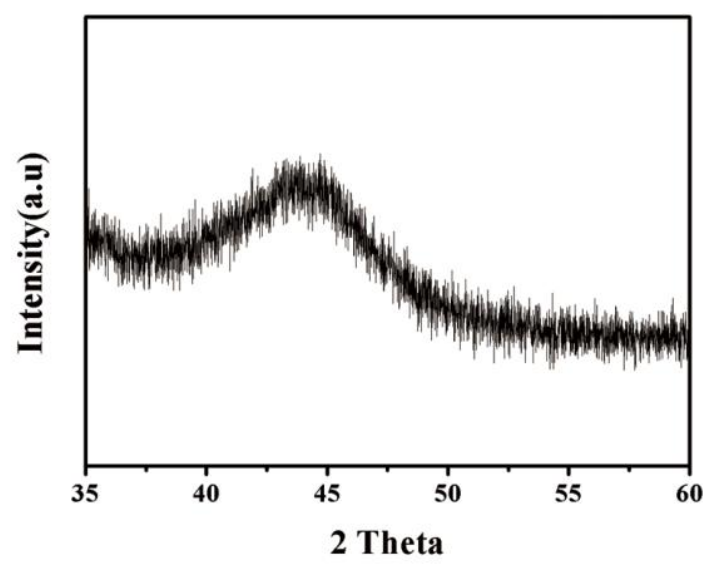

Fig.2 a typical broad diffraction pattern indicates it is amorphous phase in Ni80P20 metallic glass obviously

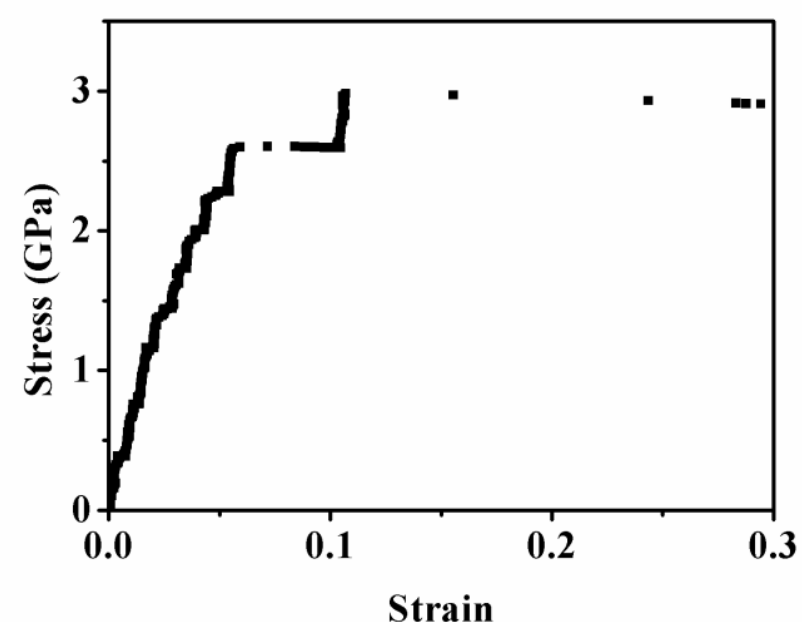

Fig.3 Stress-strain curve obtain from Nano indentation

Here micro-scale pillar compressions were tested with a diamond flat punch with Nano Indenter G200, Load-displacement date was got to analyze stress-strain curve with a constant strain rate of $10^{-3} \mathrm{~s}^{-1}$. Here, stress is calculated using Eq. (1) and strain with Eq. (2), $\mathrm{d}$ is the diameter at the top of pillar

$$
\begin{aligned}
& \text { Stress }=41 \text { load } / \pi \mathrm{d}^{2} \\
& \text { Strain }=\text { displacement } / \text { length }
\end{aligned}
$$

Fig. 3 shows the stress-strain curve of $1 \mu \mathrm{m} \mathrm{Ni}{ }_{80} \mathrm{P}_{20}$ ribbon. At the beginning of the compression, the curve is linear elastic deformation for ribbon. The ribbon's elastic modulus (young's modulus) is $50.29 \mathrm{GPa}$, the ribbon's yield strength at 0.02 strain is about $2.25 \mathrm{GPa}$. The prevailing explanation of MG deformation mechanism is shear transformation zones (STZs)[18,19]. According to this theory, STZs in MG are primary carriers of plasticity, every STZ covers hundreds atoms which located nearby. When strain from 0.1-0.45, much small burst appear which means shear band as plastic flow emission in STZs but not propagate to shear band. Beyond elastic deformation, while strain reach 5\% the big burst happen in ribbon, big burst means a main single shear band as plastic flow emission and propagate to release energy. Strain from 0.05 to 0.30 , the bursts of ribbon is 3 times, which correspond to the 3 dominating single shear bands in the pillar 
(right insert, Fig.4b) This phenomenon confirms that the combination of morphology and stressstrain curves can deduce the nature of deformation.
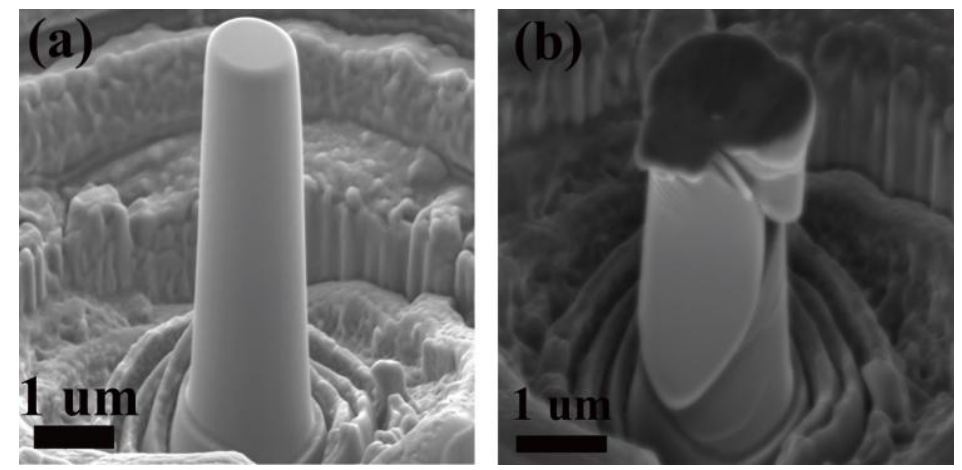

Fig.4 $1 \mu \mathrm{m}$ pillars before and after compression, (a) pillar before compression, (b) compressed pillar (strain at 0.3 ).

The SEM figures of $1 \mu \mathrm{m}$ pillars before and after compression were shown in Fig. 4. And from Fig. $4 \mathrm{~b}$, the two shear bands from the topside corner of the pillar, where is the connection between the pillar and diamond punch, the cross section area is minimum so the stress is maximal. Deformation of plastic flow located in STZs causes single main shear bands at 45 degree with the same orientation generate and propagate rapidly, deviate from original location. The detector of Nano Indenter G200 records data every $0.04 \mathrm{~s}$, the sparse dots after 0.06 strain of ribbon reveals shear band in STZs deform rapidly. Considering the 6 dots deformation time about $0.3 \mathrm{~s}$ from strain 0.12 to 0.3 , the main and catastrophic shear band occur too fast, that is very dangerous in materials engineering and all efforts are made to prevent this happen in the future. This work prove that nanoindentation with a flat punch is an effective method to characterize mechanical properties.

\section{Conclusion:}

Here we fabricated $\mathrm{Ni}_{80} \mathrm{P}_{20}$ ribbon with melt spinning. Mechanical property of $\mathrm{Ni}_{80} \mathrm{P}_{20}$ metallic ribbon was tested in micro-scale compression, 1 um pillar produced by FIB were characterized with Nano-Indention. Stress-strain curves together with compressed pillars morphology were analysis, Ribbon deformation mechanism may attributed to the shear transformation zones (STZs), STZs as preferential shear band nucleation sites and form shear bands, and shear bands rapid propagation cause catastrophic failure. This technique is effective to determine mechanical properties of microscale pillars.

\section{References}

[1] Klement W, JUN, Willens \& Amp R H, et al. Non-crystalline Structure in Solidified GoldSilicon Alloys [J]. Nature, 1960, 187(4740): 869-870.

[2] Inoue A, Ohtera K, Kita K, et al. New Amorphous Mg-Ce-Ni Alloys with High Strength and Good Ductility [J]. Japanese Journal of Applied Physics, 1988, 27(12): L2248-L2251.

[3] Ashby M F, Greer A L. Metallic glasses as structural materials [J]. Scripta Materialia, 2006, 54(3): 321-326.

[4] Lai Y H, Lee C J, Cheng Y T, et al. Bulk and microscale compressive behavior of a Zr-based metallic glass [J]. Scripta Materialia, 2008, 58(10): 890-893

[5] Lin T, Cheng Y Q, Shan Z W, et al. Approaching the ideal elastic limit of metallic glasses[J]. Nature Communications, 2011, 3(48):19596-19600.

[6] Kinetic Theory of Liquids. Oxford, at the Clarendon Press, 1946. - 360 pp. 
[7] Xing, L. Q., et al. "Enhanced plastic strain in Zr-based bulk amorphous alloys." Physical Review B 64.18(2001):607-611.

[8] Greer J R, Oliver W C, Nix W D. Size dependence of mechanical properties of gold at the micron scale in the absence of strain gradients[J]. Applied Physics A, 2005, 80(8):1625-1629.

[9] Schuster B E, Wei Q, Ervin M H, et al. Bulk and microscale compressive properties of a Pdbased metallic glass[J]. Scripta Materialia, 2007, 57(6):517-520.

[10] Bharathula A, Lee S W, Wright W J, et al. Compression testing of metallic glass at small length scales: Effects on deformation mode and stability[J]. Acta Materialia, 2010, 58(17):5789-5796.

[11] Volkert C A, Donohue A, Spaepen F. Effect of sample size on deformation in amorphous metals[J]. Journal of Applied Physics, 2008, 103(8):083539-083539-6.

[12] Puretz, J., Orloff, J., Swanson, L., 1984. Application of focused ion beams to electron beam testing of integrated circuits. Proceedings of SPIE - The International Society for Optical Engineering, vol. 471, pp. 38-46.

[13] Orloff, J., Swanson, L., Utlaut, M., Utlaut, M.W., 2002. High resolution focused ion beams: FIB and its applications: The physics of liquid metal ion sources and ion optics and their application to focused ion beam technology. Kluwer Academic Publishers. 316 pp.

[14] Thompson, K, et al. "In situ site-specific specimen preparation for atom probe tomography. " Ultramicroscopy 107.2-3(2007):131-139.

[15] Schuh, Christopher A. "Nanoindentation studies of materials." Materials Today 9.5(2006):3240.

[16] MLAPharr, W. C. Oliverand G. M. "Measurement of hardness and elastic modulus by instrumented indentation: Advances in understanding and refinements to methodology." Journal of Materials Research 19.1(2004):3-20.

[17] Fischer-Cripps, A. C. "Critical review of analysis and interpretation of nanoindentation test data." Surface \& Coatings Technology 200.14-15(2006):4153-4165.

[18] Spaepen, Frans. "On the fracture morphology of metallic glasses." Acta Metallurgica 23.5(1975):615-620.

[19] Schuh, Christopher A., T. C. Hufnagel, and U. Ramamurty. "Mechanical behavior of amorphous alloys." Acta Materialia 55.12(2007):4067-4109. 